\title{
VALOR DEL RENOGRAMA ISOTÓPICO DIURÉTICO EN EL SEGUIMIENTO DE LA VIAA URINARIA SUPERIOR TRAS CISTECTOMÍA Y DERIVACIÓN URINARIA CON INTESTINO
}

\author{
J.L. MOYANO CALVO, S. GÓMEZ BENÍTEZ*, T. CAMBIL MOLINA**, \\ J. CASTIÑEIRAS FERNÁNDEZ
}

\begin{abstract}
Servicio de Urología del Hospital Universitario Virgen Macarena de Sevilla. Fundación Joaquín Albarrán *Servicio de Radiología del Hospital San Lázaro de Sevilla.

**Servicio de Medicina Nuclear del Hospital Universitario Virgen Macarena de Sevilla.
\end{abstract}

Actas Urol Esp. 27 (8): 594-604, 2003

\section{RESUMEN}

VALOR DEL RENOGRAMA ISOTÓPICO DIURÉTICO EN EL SEGUIMIENTO DE LA VÍA URINARIA SUPERIOR TRAS CISTECTOMÍA Y DERIVACIÓN URINARIA CON INTESTINO

INTRODUCCIÓN: En nuestra experiencia es frecuente la dilatación de la vía urinaria superior tras la derivación urinaria con intestino. Para ahorrar actitudes terapéuticas innecesarias y distinguir que vías están dilatadas funcionalmente y cuales secundarias a estenosis ureterointestinal hemos utilizado el renograma isotópico diurético.

MATERIAL Y MÉTODO: 26 pacientes a los que se les ha realizado derivación intestinal bien tipo Mainz II bien tipo Paduana. Realizamos ecografỉa abdominal, urografía intravenosa, renograma diurético y urografía intravenosa.

RESULTADOS: El seguimiento oscila entre 1 y 4 años. El 54\% de las vías tras derivación están dilatadas, de las cuales sólo el 39,3\% muestran patrón obstructivo en el renograma diurético, confirmándose la estenosis en el manejo endourológico de la misma. Las vías urográficamente normales son normales o responden a diurético en el renograma. El 75\% de las vías anuladas funcionalmente en el urograma están anuladas en el renograma.

Los resultados se mantienen a lo largo del seguimiento de estos enfermos.

CONCLUSIONES: El renograma diurético es útil para distinguir que vía dilatada está obstruida de cual no lo está.

Los resultados se mantienen en el tiempo.

PALABRAS CLAVE: Renograma diurético. Neovejiga intestinal. Tracto Urinario Superior. Estenosis.

\section{ABSTRACT}

DIURETIC ISOTOPIC RENOGRAM IN THE FOLLOW-UP OF THE UPPER URINARY TRACT AFTER INTESTINAL URINARY DIVERSION

INTRODUCTION: In our experience we often observe urinary upper tract dilatation after urinari diversion with bowel. To spare uuesful approaches and therapeutics we have used diuretic renogram.

MATERIAL AND METHOD: 26 patients with urinary diversion (Mainz II or Paduana ileal neobladder). We performance ultrasound, urography and diuretic renogram.

RESULTS: Follow-up is between 1 y 4 years. After urinary diversion, 54\% of the upper tracts are dilated but only $39,3 \%$ of them are obstructed in the diuretic renogram. Normal urographic tracts are normal or have good response in the diuretic renogram. Results goes on in the time.

CONCLUSIONS: Diuretic renogram is a useful tool in the diagnosis of upper urinary tract obstruction after urinary diversion.

KEY WORDS: Diuretic renogram diuretic. Ileal neobladder. Upper Urinary Tract. Stenosis. 
$\mathrm{T}$ ras realizar una cistectomía radical y una derivación urinaria con intestino no es infrecuente la dilatación de la vía urinaria superior. Es importante poder distinguir si esta dilatación es secundaria a una estenosis de la unión ureterointestinal, con obstrucción al tránsito del bolo urinario y con lesión del parénquima renal o bien es de carácter funcional y no va a tener repercusión sobre el parénquima renal. No podemos olvidar que dilatación no es sinónimo de obstrucción.

Para poder distinguir si una vía urinaria dilatada esta obstruida o no disponemos de tres técnicas diagnósticas con distinto grado de invasividad y morbilidad:

- Estudios presión flujo

- Urografía Intravenosa (UIV) diurética

- Uso de radioisótopos

\section{A) Estudios presión flujo}

Los estudios presión flujo se basan en el análisis de las variaciones de la presión piélica renal ante un flujo urinario determinado por el investigador y con control de la presión vesical. Es necesario realizar una nefrostomía percutánea para poder acceder al sistema urinario superior y cateterizar el aparato urinario inferior. Estos test son reproducibles y no se ven afectados por la Tasa de Filtración Glomerular (TFG).

Han sido descritos por Whitaker y Pfister ${ }^{1,2}$, quienes establecen unos valores de presión que permiten determinar en la mayoría de los casos sin el sistema esta obstruido o no. Así para un flujo urinario de $10 \mathrm{ml}$ por minuto, Whitaker establece los siguientes límites:

Presión <15 cm de agua: no obstruido

Presión $>22 \mathrm{~cm}$ de agua: vía obstruida

Los valores intermedios son equívocos, pero en su experiencia estos casos no son más del $4 \%{ }^{3}$.

Pfister establece unos valores menores al restar la presión vesical:

Presión menor de $12 \mathrm{~cm}$ de agua: vía no obstruida

Presión mayor de $15 \mathrm{~cm}$ de agua. vía obstruida

Los valores intermedios son equívocos

B) Urografia Intravenosa Diurética

La UIV es muy útil para diagnosticar la obstrucción aguda de la vía, pero sin embargo su uti- lidad es limitada ante una obstrucción crónica. Es más, una UIV normal no nos descarta una obstrucción intermitente.

Para mejorar la capacidad diagnóstica de la UIV se utiliza un diurético administrado entre 5 a 10 minutos después del medio de contraste, considerándose diagnóstico de obstrucción:

Un aumento del área pélvica renal del $22 \%$.

Aparición de dolor en el flanco característico ${ }^{4}$.

\section{C) Uso de radioisótopos}

Para distinguir si la vía urinaria dilatada está obstruida o no disponemos de dos técnicas no excluyentes entre sí:

c.1. Medición del tiempo de tránsito parenquimatoso (TTP)

Se basa en la capacidad de separar mediante técnicas de deconvolución la radioactividad emitida en el área renal por el parénquima y la emitida por la pelvis renal. Un TTP alargado se correspondería con una obstrucción de la vía urinaria ${ }^{5}$.

c.2 Renograma diurético con MAG-3

Se basa en la administración de un diurético (Furosemida) bien a los 20 minutos de administración del radioisótopo $(\mathrm{F}+20)$, bien 15 minutos antes de su administración si existe afectación de la función renal (F-15) ${ }^{6-8}$. Ello permite valorar la respuesta de dos maneras:

- Mediante la morfología de la curva del renograma, lo que nos permite distinguir cinco tipos de curvas (Fig. 1):

- Renograma normal: Tipo I

- No respuesta al diurético manteniendo una tercera fase en meseta o ascendente: Tipo II (Patrón obstructivo)

- Normalización de la tercera fase de la curva del renograma

- De forma brusca: Tipo III (No obstructiva)

- De manera paulatina: Tipo IV (Equivoca)

- Descompensación tardía: doble bolo por reflujo vésicoureteral: Tipo $\mathrm{V}$

- Mediante la determinación del tiempo medio de lavado del trazador:

- No obstruido: menor de 10 minutos

- Obstruido: mayor de 20 minutos

- Equívoco: entre estos dos valores 


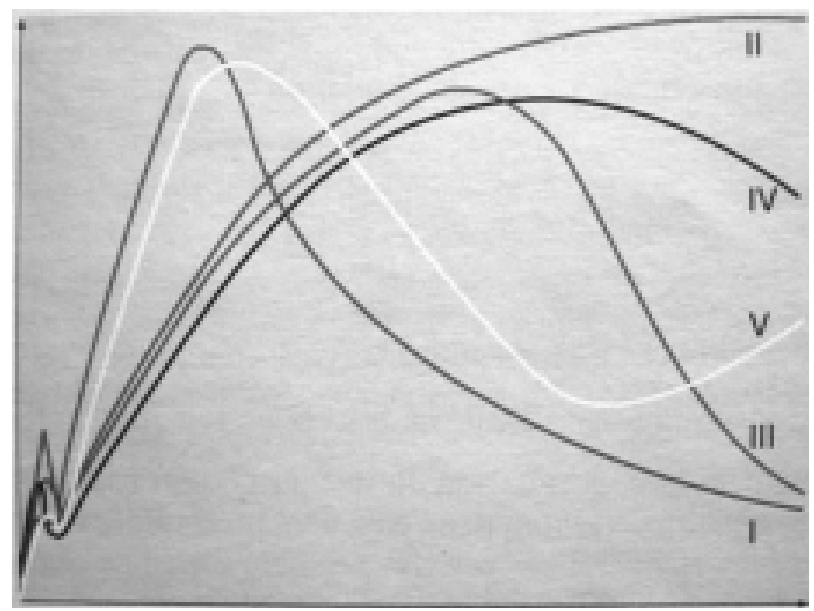

FIGURA 1. Clasificación de O'Reilly.

Las funciones renales se valoran dibujando áreas de interés en la cortical, excluyendo el sistema excretor.

En nuestro Servicio hemos optado por la utilización del renograma isotópico diurético. Los resultados que hemos obtenido son el motivo del presente trabajo.

\section{MATERIAL Y MÉTODO}

Tras la realización de una cistectomía radical practicamos una derivación urinaria con intestino, bien Mainz II, bien neovejiga ileal tipo Paduana. Los enfermos son luego seguidos en la consulta externa de neoplasias vesicales, siendo citados al mes del alta hospitalaria.

Desde finales de 1998 en el seguimiento de la vía urinaria superior realizamos las siguientes pruebas:

- Ecografía renal y vesical (en neovejigas tipo paduana)

- Urografía Intravenosa

- Renograma isotópico con test de Furosemida

Inicialmente se planeó realizar en el primer año revisiones cuatrimestrales. Tras la experiencia con los primeros enfermos y al ver la evolución de los mismos se modificó el esquema reduciendo el número de estudios urográficos y con radioisótopos, como se comentará en la discusión.

En el presente estudio valoramos:

- el estado de la vía urinaria pre-cirugía mediante UIV
- el estado post-cirugía mediante UIV

- los resultados del renograma isotópico diurético

Llevamos realizados veintiséis pacientes con un seguimiento mínimo de un año, oscilando entre cuatro y uno.

\section{RESULTADOS}

Se muestran en las siguientes tablas.

Las Tablas I y II, muestran los resultados tanto de la urografía previa como la realizada posderivación urinaria. Como podemos apreciar en la tabla 2, el 54\% de las vías muestran dilatación post-derivación, y un $8 \%$ están anuladas funcionalmente, mientras que previa a la derivación sólo estaban dilatadas el 35\%.

El comportamiento de la vía urinaria en el renograma diurético se presenta en la Tabla III, estando obstruidas el $23 \%$ de las vías urinarias y con función renal anulada el $6 \%$.

\section{TABLA I}

ESTADO DE LA VÍA URINARIA, EVALUADO POR UROGRAFÍA INTRAVENOSA ANTES DE REALIZAR LA CIRUGÍA

\begin{tabular}{|c|c|c|}
\hline & Casos & $\%$ \\
\hline Normal & 34 & 65 \\
\hline Dilatada & 18 & 35 \\
\hline
\end{tabular}

TABLA II

ESTADO DE LA VÍA URINARIA EVALUADA POR UROGRAFÍA INTRAVENOSA TRAS REALIZAR LA DERIVACIÓN URINARIA

\begin{tabular}{|c|c|c|}
\hline & Casos & $\%$ \\
\hline Normal & 20 & 38 \\
\hline Dilatada & 28 & 54 \\
\hline Anulada & 4 & 8 \\
\hline
\end{tabular}

TABLA III

COMPORTAMIENTO DE LA VÍA EN EL RENOGRAMA ISOTÓPICO DIURÉTICO

\begin{tabular}{|c|c|c|}
\hline & Casos & $\%$ \\
\hline Normal & 13 & 25 \\
\hline Dilatada no obstruida & 24 & 46 \\
\hline Dilatada obstruida & 12 & 23 \\
\hline Anulada & 3 & 6 \\
\hline
\end{tabular}


La función renal se muestra en la Tabla IV, el $56 \%$ de las unidades renales mantienen una función renal normal, mientras que disminuye en un $38 \%$ y está anulada en un $6 \%$.

\section{TABLA IV}

FUNCIÓN RENAL EN EL RENOGRAMA ISOTÓPICO

\begin{tabular}{|c|c|c|}
\hline & Casos & $\%$ \\
\hline Normal & 29 & 56 \\
\hline Disminución & 20 & 38 \\
\hline Anulada & 3 & 6 \\
\hline
\end{tabular}

Como podemos apreciar en la Tabla V el 62\% de las vías urinarias que en la UIV prederivación eran normales están dilatadas o anuladas, normalizándose el 35,3\% de las vías previamente dilatadas.

En la Tabla VI podemos apreciar:

- Todas las vías que urográficamente son normales, o bien su comportamiento es normal o bien responden a la administración de Furosemida en el renograma diurético, lo que corresponderían a curvas tipo I o IIIa del esquema de O`Reilly.

- De las vías urográficamente dilatadas postderivación están obstruidas el 39,3\% de las mismas (Curva II de O`Reilly).

- De las vías con anulación funcional urográfica post-derivación están obstruidas el 25\%.

Como podemos apreciar en la Tabla VII, el 90\% de las UIV normales post-derivación tiene una función normal en el renograma, mientras que sólo son normales el 39,3\% de las que muestran dilatación de vía y ninguna de las que están anuladas funcionalmente.

El 60,7\% de las vías dilatadas tienen disminución de la función renal y el $75 \%$ de las vías que presentan anulación funcional urográfica, tienen anulación en el renograma.

Tal como muestra la Tabla VIII, el 92\% de las unidades que eliminan espontáneamente tienen una función renal normal, así como el 54,2\% de las que eliminan tras la administración de diurético. Como era de esperar las vías que están obstruidas y no responden a diurético tienen afectada la función con mayor frecuencia, 66,7\% de los casos, pero un $33,3 \%$ siguen teniendo función renal normal

TABLA V

COMPARACIÓN ENTRE EL ESTADO DE LA VÍA EN LA UIV PRE Y POST DERIVACIÓN

\begin{tabular}{|c|c|c|c|c|c|}
\hline & & & t derivac & & \\
\hline \multirow{5}{*}{ Pre derivación } & & & Normal & Dilatada & Anulada \\
\hline & \multirow{2}{*}{ Normal } & Casos & 13 & 19 & 2 \\
\hline & & $\%$ & $38 \%$ & $56 \%$ & $6 \%$ \\
\hline & \multirow{2}{*}{ Dilatada } & Casos & 6 & 10 & 1 \\
\hline & & $\%$ & $35,3 \%$ & $58,8 \%$ & $5,9 \%$ \\
\hline
\end{tabular}

TABLA VI

COMPARACIÓN ENTRE EL ESTADO DE LA VÍA EN LA UROGRAFÍA POST-DERIVACIÓN Y EL COMPORTAMIENTO DE LA VÍA EN EL RENOGRAMA ISOTÓPICO DIURÉTICO POST-DERIVACIÓN

\begin{tabular}{|c|c|c|c|c|c|c|}
\hline \multicolumn{7}{|c|}{ Renograma post-derivación } \\
\hline \multirow{7}{*}{$\begin{array}{l}\text { UIV } \\
\text { post-derivación }\end{array}$} & & & Normal & $\begin{array}{c}\text { Dilatada no } \\
\text { obstruida }\end{array}$ & $\begin{array}{c}\text { Dilatada } \\
\text { obstruida }\end{array}$ & Anulada \\
\hline & \multirow{2}{*}{ Normal } & Casos & 12 & 8 & & \\
\hline & & $\%$ & $60 \%$ & $40 \%$ & & \\
\hline & \multirow{2}{*}{ Dilatada } & Casos & 1 & 16 & 11 & \\
\hline & & $\%$ & $3,6 \%$ & $57,1 \%$ & $39,3 \%$ & \\
\hline & \multirow{2}{*}{ Anulada } & Casos & & & 1 & 3 \\
\hline & & $\%$ & & & $25 \%$ & $75 \%$ \\
\hline
\end{tabular}


TABLA VII

RELACIÓN ENTRE EL ESTADO DE LA VÍA EN LA UIV POST-DERIVACIÓN Y LA FUNCIÓN RENAL EN EL RENOGRAMA ISOTÓPICO POST-DERIVACIÓN

\begin{tabular}{|c|c|c|c|c|c|}
\hline & & & 1a post-c & & \\
\hline \multirow{7}{*}{$\begin{array}{l}\text { UIV } \\
\text { post-derivación }\end{array}$} & & & Normal & Disminución & Anulada \\
\hline & \multirow{2}{*}{ Normal } & Casos & 18 & 2 & \\
\hline & & $\%$ & $90 \%$ & $10 \%$ & \\
\hline & \multirow{2}{*}{ Dilatada } & Casos & 11 & 17 & \\
\hline & & $\%$ & $39,3 \%$ & $60,7 \%$ & \\
\hline & \multirow{2}{*}{ Anulada } & Casos & & 1 & 3 \\
\hline & & $\%$ & & $25,0 \%$ & $75,0 \%$ \\
\hline
\end{tabular}

TABLA VIII

RELACIÓN EN EL RENOGRAMA ENTRE EL COMPORTAMIENTO DE LA VÍA Y LA FUNCIÓN RENAL

\begin{tabular}{|c|c|c|c|c|c|}
\hline \multicolumn{6}{|c|}{ Función renal } \\
\hline \multirow{9}{*}{$\begin{array}{l}\text { Comportamiento } \\
\text { vía }\end{array}$} & & & Normal & Disminución & Anulada \\
\hline & \multirow{2}{*}{ Normal } & Casos & 12 & 1 & \\
\hline & & $\%$ & $92 \%$ & $8 \%$ & \\
\hline & \multirow{2}{*}{$\begin{array}{c}\text { Dilatada no } \\
\text { obstruida }\end{array}$} & Casos & 13 & 11 & \\
\hline & & $\%$ & $54,2 \%$ & $45,8 \%$ & \\
\hline & \multirow{2}{*}{$\begin{array}{c}\text { Dilatada } \\
\text { obstruida }\end{array}$} & Casos & 4 & 8 & \\
\hline & & $\%$ & $33,3 \%$ & $66,7 \%$ & \\
\hline & \multirow{2}{*}{ Anulada } & Casos & & & 3 \\
\hline & & $\%$ & & & $100,0 \%$ \\
\hline
\end{tabular}

\section{DISCUSION}

Los estudios con radioisótopos tienen una serie de ventajas en comparación con los que utilizan medios de contraste iodados:

- Mínima invasividad

- No precisan preparación del enfermo

- No existen reacciones alérgicas

- Menor radiación que con RX

- No son nefrotóxicos

- Son funcionales y cuantitativos

- Permiten manipulación farmacológica

En el estudio funcional de la vía urinaria superior, el renograma isotópico con test de Furosemida tiene una correlación igual o superior al $85 \%$ con el test de Whitaker ${ }^{9}$. Tiene una tasa de falsos positivos menor de 10\%, fundamentalmente por

- Tipo obstrucción

- Función renal

- Tamaño pelvis renal

- Tiempo administración diurético
- Grado repleción de la vejiga

- Grado de hidrataciónUn sistema dilatado no tiene por qué ser un sistema obstruido, y aunque la urografía intravenosa procura una adecuada información anatómica, no es capaz de distinguir entre un sistema obstruido de uno que no lo está.

Por ello y por las ventajas ya señaladas de los estudios con radioisótopos, nos planteamos en nuestro Servicio la realización de renograma isotópico con test de Furosemida en todos los enfermos a los que practicamos derivación urinaria con intestino.

En la literatura consultada, no hemos encontrado ningún estudio que haga referencia al uso del renograma isotópico con test de Furosemida para seguimiento de la vía urinaria superior en este tipo de enfermos. Sólo hemos encontrado dos estudios que lo utilizan para valorar el comportamiento de la vía ante la repleción del reservorio, pero no como estudio de la vía en sí misma $^{16,19}$. 
- Sandor ${ }^{10}$ en 1966 compara renograma y urografía intravenosa en 350 enfermos con dilatación de la vía urinaria superior. Concluyó que el renograma confirmaba o aportaba nuevos datos a los estudios urográficos, y que estos datos eran útiles en el enfoque terapéutico del enfermo. El renograma debía utilizarse en conjunto con el resto de las pruebas.

- Ballarati y Duncelli ${ }^{11}$ comparan urografía intravenosa y renograma en 39 pacientes con neoplasia vesical. En el 60\% de los casos el resultado de ambas pruebas era concordante, discrepando en el resto de los casos, en todos ellos, la cistoscopia y otras pruebas renales confirmaban los resultados renográficos, por lo que concluían que le renograma era más sensible y fiable.

- Soloway ${ }^{12}$ en 1972 compara renograma y urografía intravenosa en 30 pacientes con conducto ileal con un seguimiento medio de 6,7 años. Observa que un renograma normal se corresponde con una pielografía normal. Aquellos casos con caliectasia o pieloectasia tenían un T máx. alargado siendo la diferencia significativa (2,94 minutos en sistemas normales, 4,62 en sistemas mínimamente dilatados, 6,32 con dilatación moderada y más de 20 minutos en sistemas muy dilatados). Así mismo observa que la sensibilidad del renograma a cambios en el flujo urinario lo hacen particularmente útil para evaluar la obstrucción del tracto urinario superior. Así mismo su seguridad, ventajas y exactitud lo hacen ideal para el seguimiento a largo plazo de los pacientes.

- Wilson ${ }^{13}$ en 1977 compara renograma y urografía intravenosa en 35 pacientes a los que realiza ureterosigmoidostomía. Realiza ambos estudios pre y post-operatorios. En le preoperatorio existe una buena correlación entre ambos estudios, mientras que en le postoperatorio el $50 \%$ de las vías urográficamente normales mostraban renogramas alterados, bien en la fase de tránsito, bien en la de eliminación. La mayoría de estas alteraciones eran leves sin empeoramiento en sucesivos estudios. Todas las vías urinarias dilatadas en la urografia presentaban renogramas claramente alterados. Concluye que el seguimiento de este tipo de enfermos, puede realizarse con renograma, analítica y radiografía simple de aparato urinario, reservando la urografía para aquellos casos con alteraciones en el renograma.
- Akerlund ${ }^{14}$ en 1987 observa que el grado de repleción del reservorio influye en el comportamiento del aparato urinario superior, a menor presión del reservorio mejor drenaje y función renal.

- Chen ${ }^{15}$ en 1991 realiza renogramas de forma preoperatoria, al mes y cada seis meses durante dos años y medio a 61 pacientes a los que realiza una bolsa de Kock. Observa que al mes se produce una disminución de la función renal en todos los pacientes, con posterior estabilización de la misma. Las unidades renales con hidronefrosis muestran un T máx. Mayor y alteraciones en la fase de eliminación el renograma.

- Takeda ${ }^{16}$ en 1993 realiza renograma diurético a 14 pacientes a los que realiza bien una cistoplastia de aumento bien derivación urinaria. Valora como influye el grado de repleción del reservorio en el renograma, observando que el $85 \%$ de las vías muestran una curva normal con reservorio vacío. En aquellos pacientes (9/14) que no presentan ondas de presión superiores a $20 \mathrm{~cm}$ de H2O en el reservorio, la repleción del mismo no influye en el renograma, mientras que en el resto (5/14) con presiones superiores a esa cifra sí se altera el renograma con la repleción del reservorio.

- Kato ${ }^{17}$ en 1994 estudia 14 pacientes a los que ha derivado mediante una vejiga rectal ileocecal con renograma isotópico, observando que el $68 \%$ de las vías son normales, el 25\% están dilatadas pero no obstruidas y el $7 \%$ restante dilatadas y obstruidas. Al año cinco de las vías dilatadas no obstruidas e han normalizado, y todas las que eran normales lo siguen siendo. Concluye que el renograma isotópico ayuda a evitar cirugías o maniobras terapéuticas invasivas en las vías dilatadas.

- Kristjánsson ${ }^{18}$ en 1995 compara 56 pacientes con 16 años de seguimiento medio a los que ha realizado conducto ileal, colónico o reservorio continente. Observa una disminución de la tasa de filtración glomerular (TFG) superior al $25 \%$ en el 28\% de los enfermos, sin que influya el tipo de anastomosis realizada. El T máx. En el renograma era superiora 4,5 minutos en el $50 \%$ de las vías urinarias, pero al valorar el tiempo de tránsito parenquimatoso sólo 13 vías presentaban resultados dudosos. La mitad e las vías con tiempo de tránsito alargado y alteración de la TFG presentaban estenosis de la unión ureterointestinal. 
- Okuno ${ }^{19}$ en el año 2000 realiza renograma diurético a 43 pacientes a los que realiza derivación bien con bolsa de Kock, bien tipo Hautmann. Su objetivo es conocer como afecta la presión del reservorio a la función del aparato urinario superior. Distingue cinco patrones: normal, alta presión, retención, obstrucción y reflujo. En conjunto 78\% de las derivaciones tipo Kock y el 60\% de las Hautmann presentan morfologías anormales en el renograma, considerando obstruidas el 5\% delas Kock y ninguna de las Hautmann.

En nuestra experiencia, el renograma isotópico diurético nos permite distinguir que vías dilatadas están obstruidas de las que no. Las imágenes se muestran en las Figuras 2 a la 13.

Sólo un $39 \%$ de las vías dilatadas tienen una causa obstructiva (hasta ahora estenosis benigna de la unión ureterointestinal), siempre antes de los cuatro meses post derivación. Un tercio de estos sistemas obstruidos mantienen función renal normal, y tras la resolución de la estenosis la función renal se mantiene estable.

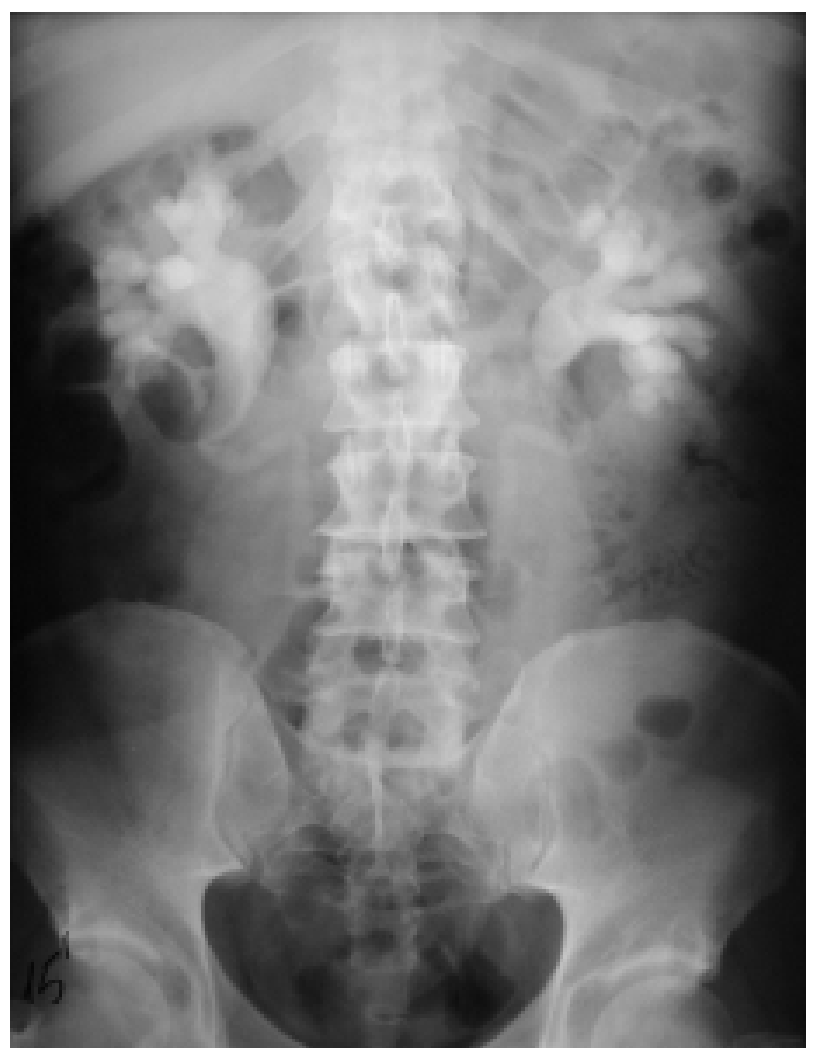

FIGURA 2. Urografia que muestra dilatación de la via urinaria bilateral.

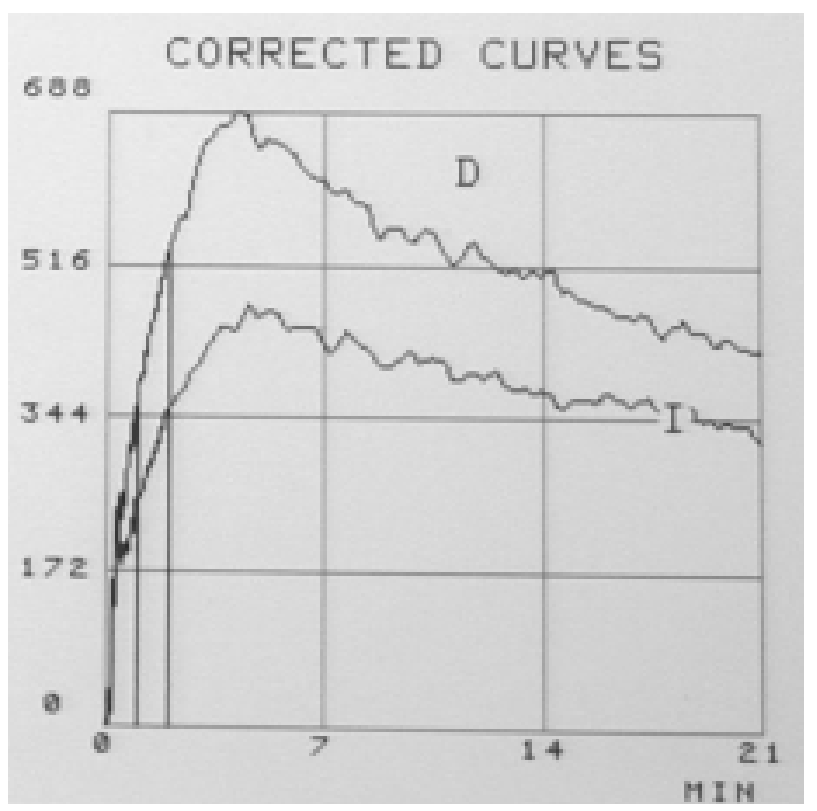

FIGURA 3. Renograma isotópico que muestra imagen "en meseta" en la fase de eliminación. Es el mismo enfermo de la Figura 2.

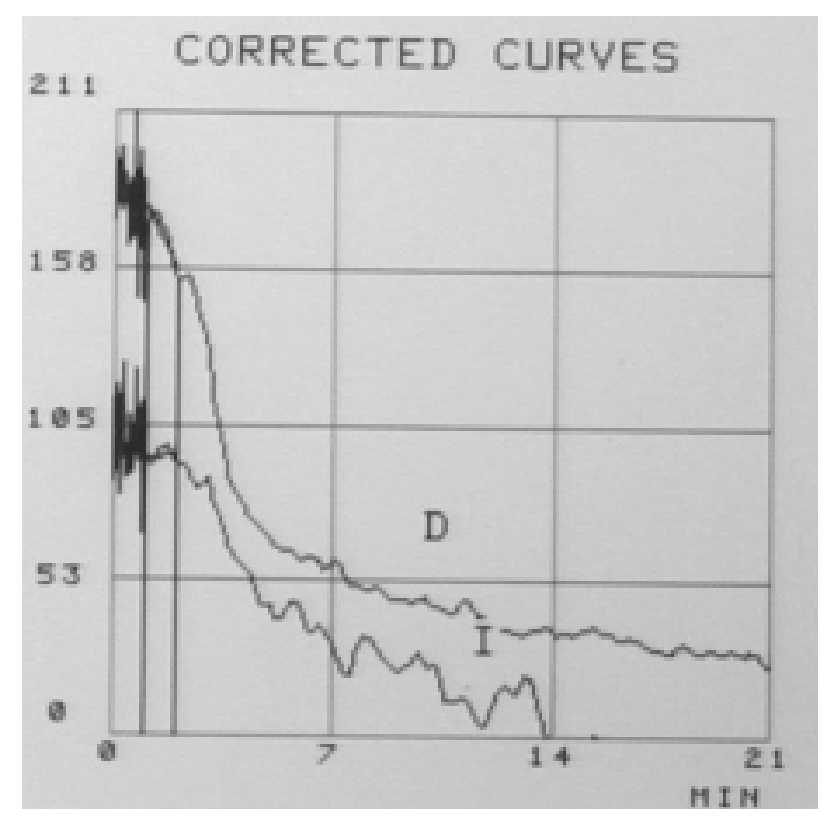

FIGURA 4. Renograma en el mismo enfermo tras administración de diurético. Rápida eliminación del radionúclido. Curva tipo III de O'Reilly.

En el seguimiento a largo plazo las vías dilatadas no obstruidas mantienen su función en los sucesivos estudios.

Inicialmente planteamos realizar renogramas cuatrimestrales durante el primer año en todos los enfermos, independientemente del estado de 


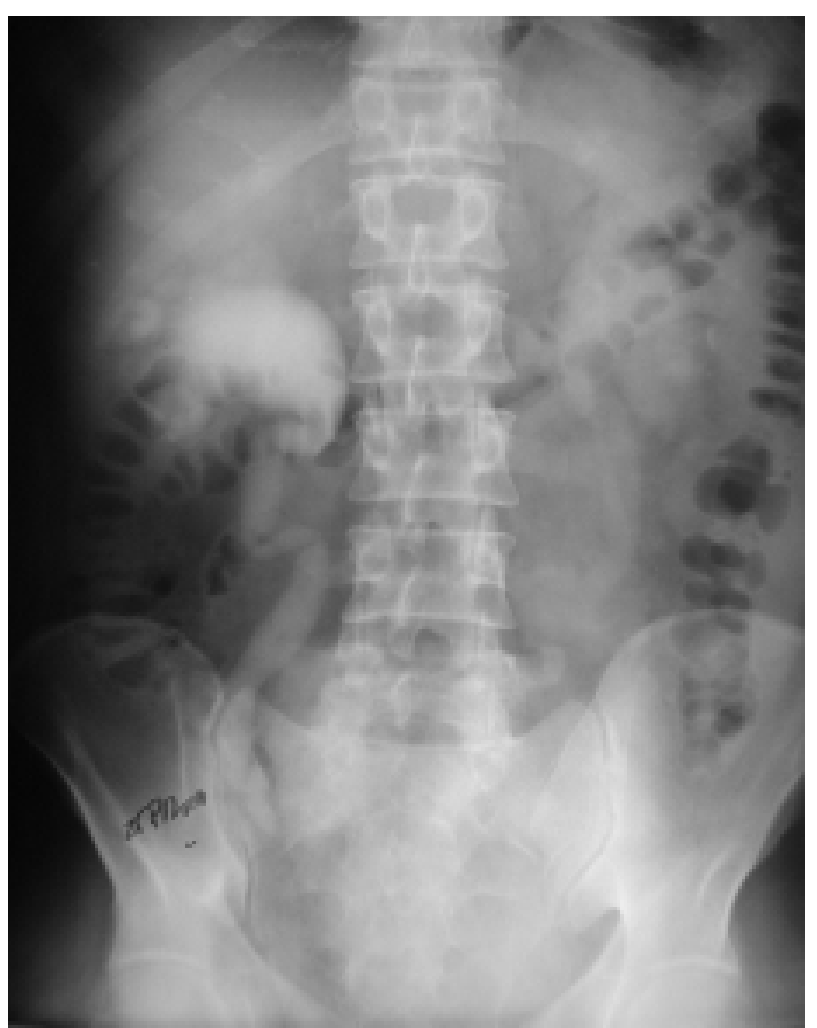

FIGURA 5. Urografia que muestra ureterohidronefrosis bilateral mayor en el lado derecho.

la vía. Posteriormente viendo que aquellos enfermos sin dilatación de la vía urinaria tenían un renograma normal y que los que mostraban vía dilatada sin obstrucción no existía variación en el renograma a lo largo de los sucesivos estudios hemos replanteado totalmente el seguimiento de la vía superior en estos enfermos:

- Primera visita post-derivación

- Historia Clínica

- Analítica con Gasometría venosa

- Ecografía Abdominal (la realizamos nosotros en la misma visita)

- Vía urinaria superior sin dilatación

- Seguimiento cuatrimestral el primer año y semestral los sucesivos con Analítica y Ecografía, a partir del quinto año seguimiento anual.

- Urografía Intravenosa anual

- Vía urinaria superior con dilatación no obstruida

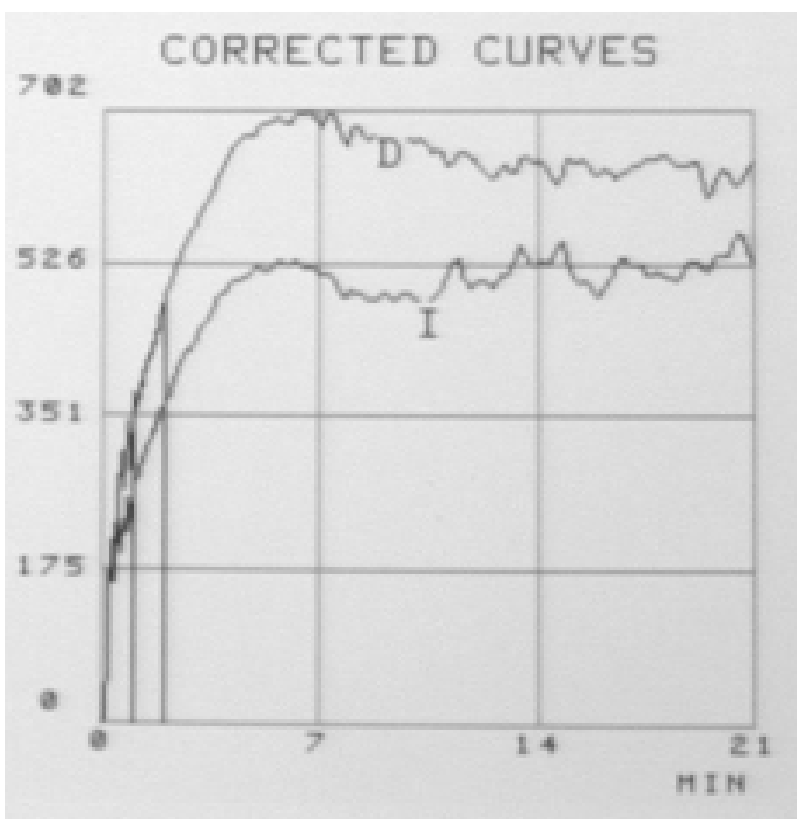

FIGURA 6. Renograma en el mismo enfermo de la Figura 5, que muestra imagen "en meseta" en la fase de eliminación.

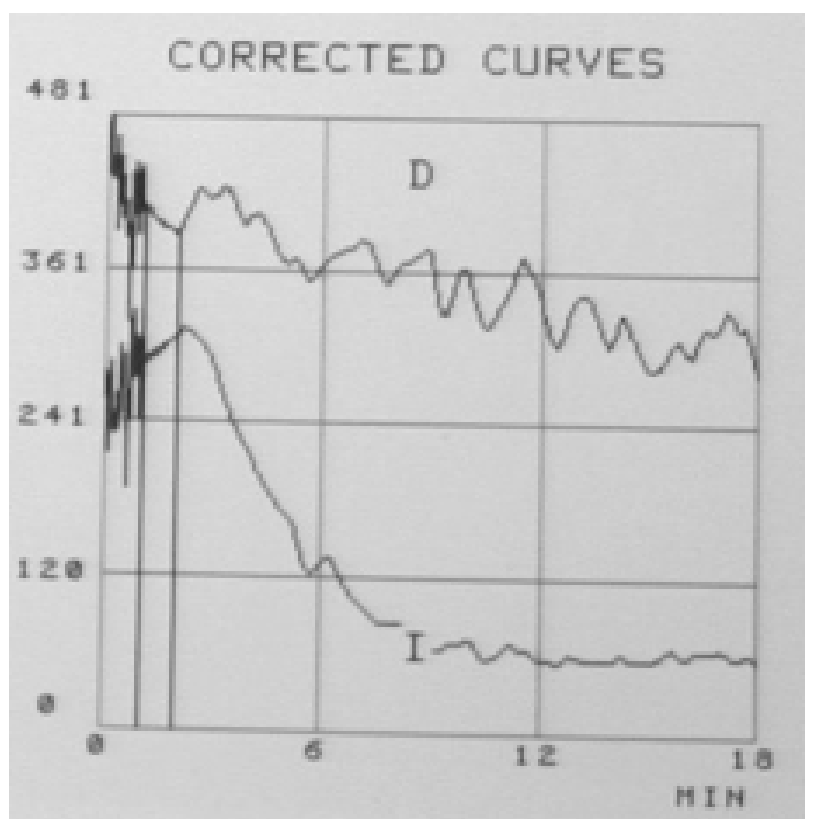

FIGURA 7. Renograma tras diurético en el mismo enfermo. Respuesta tipo III (no obstructiva) en la unidad renal izquierda y tipo II (obstructiva) en la derecha.

- Seguimiento cuatrimestral el primer año y semestral los sucesivos con Analítica y Ecografía, a partir del quinto año seguimiento anual.

- Dos renogramas isotópicos los dos primeros años

- Urografía Intravenosa anual 


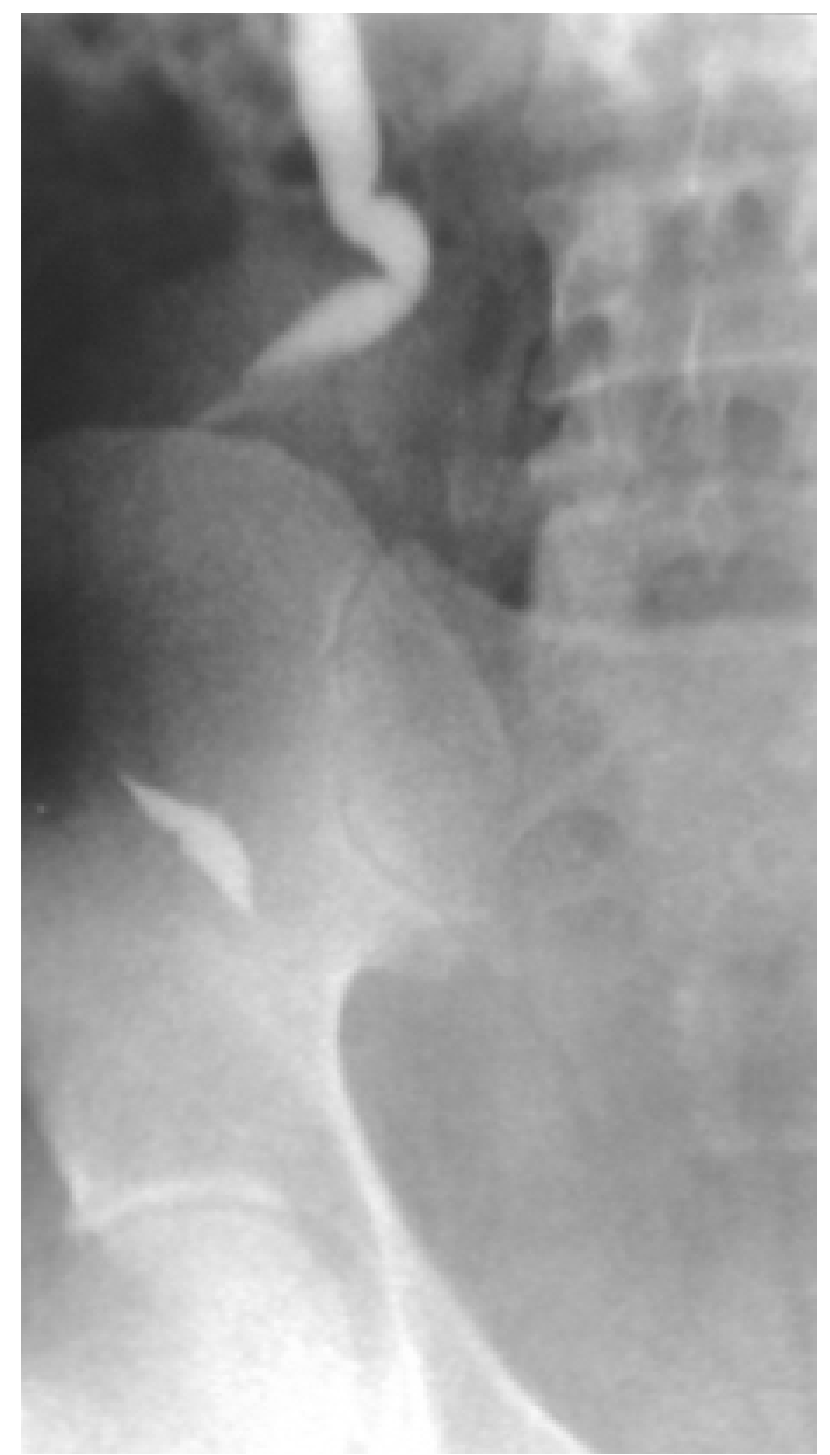

FIGURA 8. Pielografia anterógrada derecha que muestra estenosis distal derecha. Mismo enfermo que en la Figura 5

- Vía urinaria superior obstruida, tras la resolución de la misma

- Seguimiento cuatrimestral el primer año y semestral los sucesivos con Analítica y Ecografía, a partir del quinto año seguimiento anual.

- Tres renogramas isotópicos el primer y dos el segundo año

- Urografía Intravenosa anual

Posiblemente en el futuro en las vías dilatadas no obstruidas realicemos renogramas semestrales en el primer año y anual en el segundo, así mismo según veamos la evolución de las unidades a las que se les haya resuelto la obstrucción reduzcamos exploraciones.

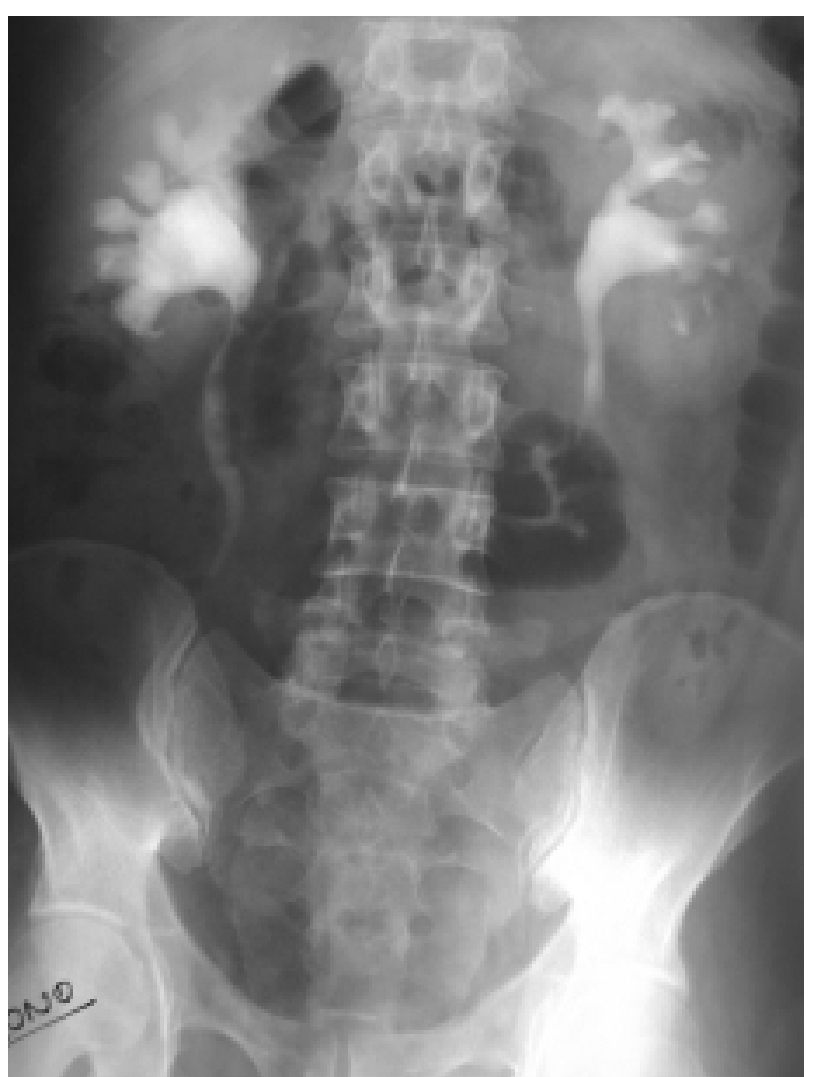

FIGURA 9. Urografia intravenosa tras la resolución endoscópica de la estenosis ureterointestinal. Mismo enfermo que en la Figura 5.

Suprimimos el renograma en vías no dilatadas ya que este no sustituye a la información anatomo-funcional y oncológica que nos proporciona el urograma intravenoso. Al no estar dilatada la vía, consideramos más razonable realizar el urograma que el renograma.

\section{CONCLUSIONES}

El renograma isotópico diurético permite distinguir aquellas vías dilatadas obstruidas de aquellas que no lo están, permitiendo ahorrar exploraciones y tratamientos invasivos no exentos de morbilidad.

$\mathrm{El}$ renograma isotópico diurético es una prueba útil en el seguimiento a largo plazo de las vías urinarias superiores, permitiendo valorar de forma cuantitativa la función de cada unidad renal por separado.

Puede ser perfectamente introducido como prueba de rutina en le seguimiento de estos enfermos, realizándolo en caso de dilatación de la vía urinaria. 


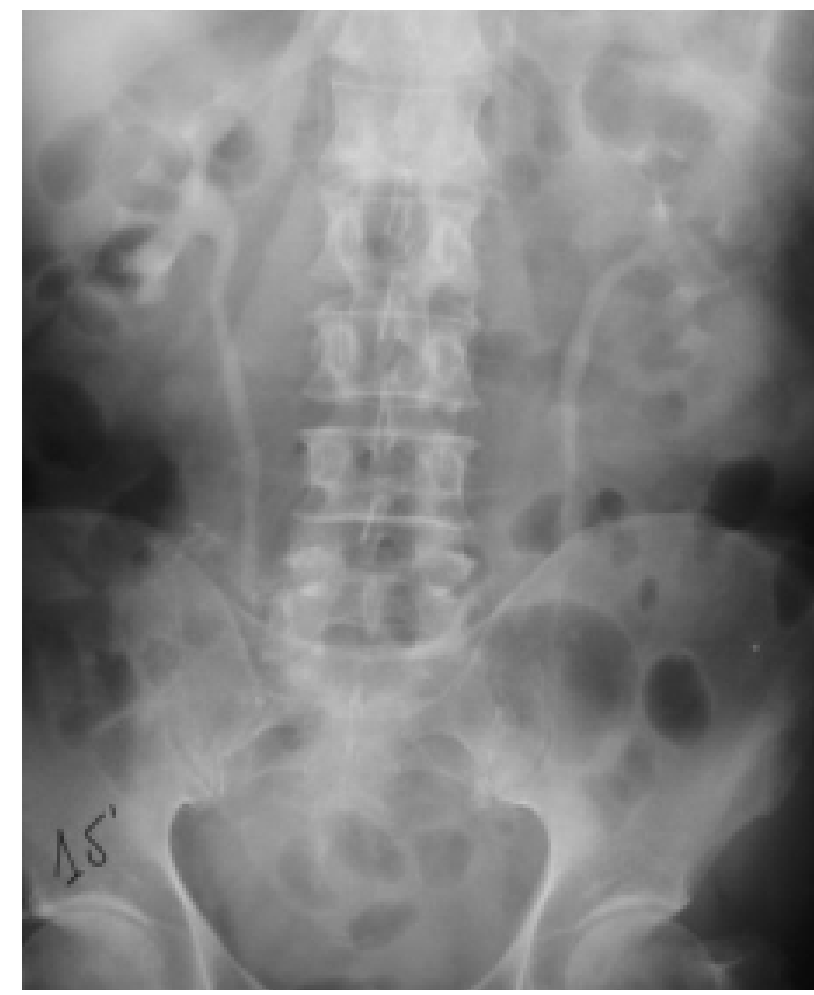

FIGURA 10. Urografia intravenosa que muestra minima ectasia de via urinaria derecha.

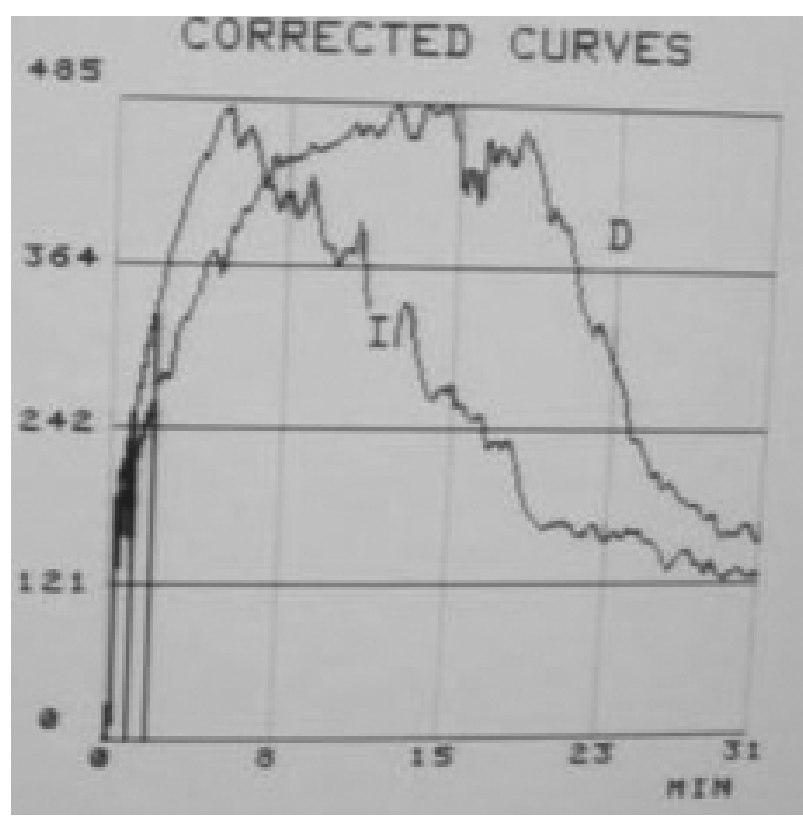

FIGURA 11. Renograma del mismo enfermo de la Figura 10 que se considera normal.

\section{REFERENCIAS}

1. WHITAKER RH.: The Whitaker Test. Urol Clin North Am 1979; 6: 529-539.

2. PFISTER RC, NEWHOUSE JH.: Intreventional percutaneous techniques. I. Antegrade pyelography and ureteral perfusion. Radiol Clin North Am 1979; 17: 341-350.

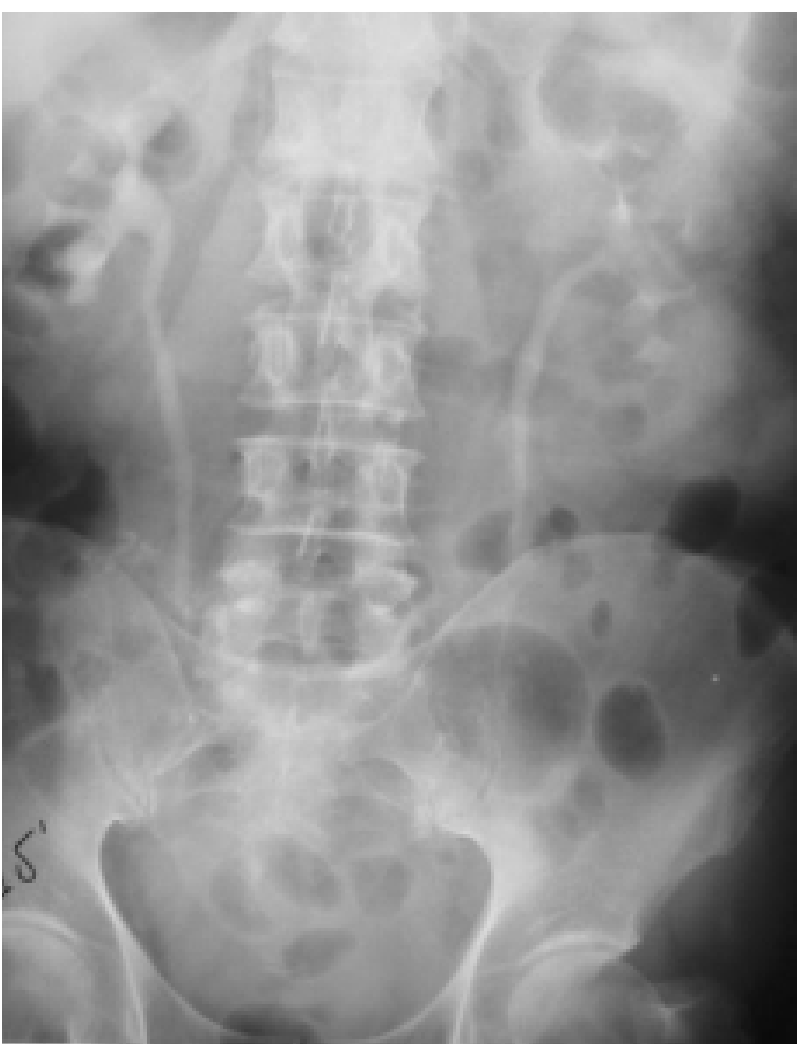

FIGURA 12. Urografia Intravenosa en el mismo enfermo que la Figura 10 un año después. No cambios.

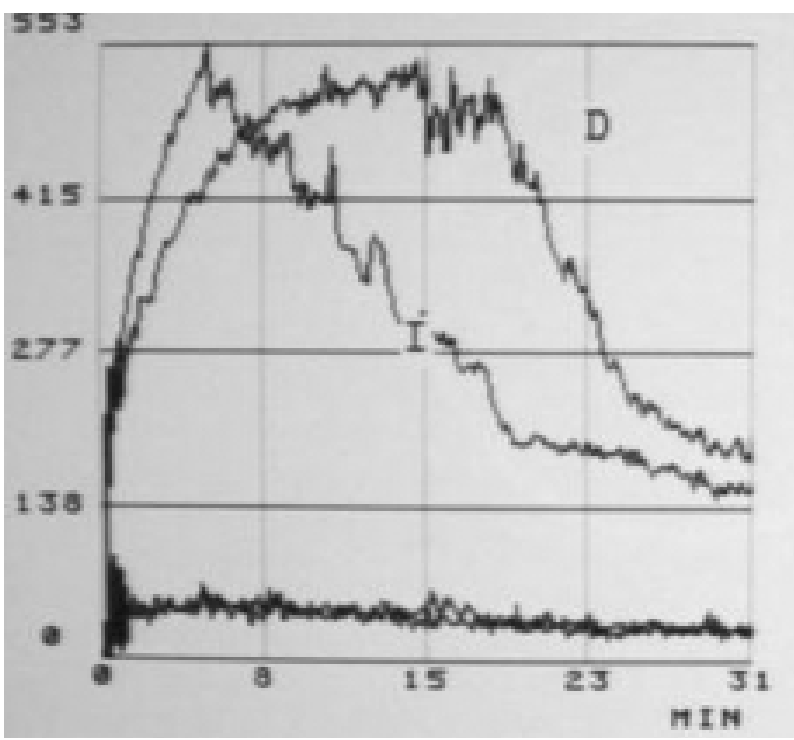

FIGURA 13. Renograma del mismo enfermo de la Figura 10 un año después. No cambios.

3. WHITAKER RH.: An evaluation of 170 diagnostic pressure-flow studies of the upper urinary trac. $\mathrm{Br} J$ Urol 1979; 121: 602-604.

4. WHITFIELD HN, BRITTON KE, HEDNRY WF, WICKHAM JEA.: Furosemide intravenous urography in the diagnosis of pelvicureteric junction obstruction. Br J Urol 1979; 51: 445-448. 
5. LAWSON RS, BROWN NGJ, DANCE DR et al.: A multicentre comparsion of techniques for deconvolution of teh renogram. Communication to the British Nuclear Medicine Society, Sevent Annual Meeting, 1979.

6. O’REILLY PH, TESTA HJ, LAWSON RS, FARRAR DJ, CHARTLON-EDWARDS E, CARROL RNP, BARNARD RJ.: Diuresis renography in equivocal urinary tract obstruction. Br J Urol 1979; 50: 76-80.

7. O`REILLY PH.: Diuresis renography 8 years later: an update. $J$ Urol 1986; 136: 993-995.

8. UPSDELL SM, LEESON SM, BROMAN PJC, O`REILLY PH.: Diuretic-induced urinary flow rates at vaying clearances and their relevance to the performance and intrepretation of duiresis renography. Br J Urol 1998; 61: 14-18.

9. KOFF SA, THRALL JH, KEYES WJ Jr.: Assesment of hydroureteronephrosis in children using diuretic radioniclide urography. $J$ Urol 1980; 123: 531534.

10. SANDOR HW, McDONALD DF.: The renogram vs. Pyelogram: evaluation of the significance of upper urinary tract obstruction. J Urol 1966; 96: 816827.

11. BALLARATI U, PURICELLI G.: Study of renal function by means of urography and radionephrography in patients with vesical neoplasms. Radiol Med (Torino) 1965; 51: 1095-1111.

12. SOLOWAY MS, MYERS GH Jr, JOHNSTON MD, KETCHAM AS.: Renography in the evaluation of patients with ileal conduits. Surg Gynecol Obstet 1972; 135: 521-524.

13. WILSON KS, DOIG A, FOWLER JW.: Renography in the assesment of patients with uretero-sigmoid diversion. Br J Urol 1977; 49: 195-198.
14. AKERLUND S, BERGLUND B, GRANERUS G, JENSEN J, KOCK NG, PHILIPSON BM.: Drainage of the upper urinary tract as recorded in patients with a continet urostomy (Kock pouch). Scand J Urol Nephrol 1987; 21: 109-114.

15. CHEN KK, CHANG LS, CHEN MT, YEH SW.: Prospective radionuclide renal function evaluation and its correlation with radiological findings in patients with Kock pouch urinary diversion. $J$ Urol 1991; 145: 952-955.

16. TAKEDA M, KATAYAMA Y, TSUTSUI $\mathrm{T}$ et al.: Evaluation of upper urinary tract dynamics by diuresis renography in patients receiing urinary reservoir operation. Urol Int 1993; 51: 117-124.

17. KATO T, SATO K, KAKINUMA H, KOBAYASHI M, TAMURA K.: Radionuclide evaluation of teh uretero-ileoceco-proctostomy ileocecal rectal bladder. Int J Urol 1994; 1: 156-161.

18. KRISTJANSSON A, WALLIN L, MANSSON W.: Renal function up to 16 years after conduit (refluxing or anti-reflux anastomosis) or continet urinary diversion. 1.Glomerular filtration rate and patency of ureterointestinal anastomosis. Br J Urol 1995; 76: 539-545.

19. OKUNO T, FRANCO CORONEL OE, YANAGAWA M, TAKEDA K, KAWAMURA J.: Effect of increased trareservoir prsesure on upper urinary tract fction in continent urinary diversion ts assesed by radioisotope renography. Utrol Int 2000; 64: 185-191.

Dr. J.L. Moyano Calvo

C/ Bogotá, 19 - portal 3, 2 B

41013 Sevilla

(Trabajo recibido el 4 marzo de 2003) 